AperTO - Archivio Istituzionale Open Access dell'Università di Torino

\title{
Leveraging MPEG-21 User Description for Interoperable Recommender Systems
}

\section{This is the author's manuscript}

Original Citation:

Availability:

This version is available http://hdl.handle.net/2318/1631991

since 2017-04-05T18:28:20Z

Publisher:

ACM

Published version:

DOI:10.1145/2851613.2851920

Terms of use:

Open Access

Anyone can freely access the full text of works made available as "Open Access". Works made available under a Creative Commons license can be used according to the terms and conditions of said license. Use of all other works requires consent of the right holder (author or publisher) if not exempted from copyright protection by the applicable law. 


\title{
Leveraging MPEG-21 User Description for Interoperable Recommender Systems
}

\author{
Sabino Metta ${ }^{1}$, Paolo Casagranda ${ }^{1,2}$, Alberto Messina ${ }^{1}$, Maurizio Montagnuolo ${ }^{1}$ and \\ Francesco Russo ${ }^{1}$ \\ ${ }^{1}$ RAI Radiotelevisione italiana, via Cavalli 6, Torino, Italy \\ ${ }^{2}$ Università di Torino, Computer Science Department, Corso Svizzera 185, Torino, Italy \\ \{sabino.metta, paolo.casagranda, alberto.messina, maurizio.montagnuolo, \\ francesco.russo\}@rai.it
}

\begin{abstract}
The paper introduces the MPEG-21 User Description standard, referred to as MPEG-21 UD, aimed at enabling the horizontal integration of recommendations coming from different service providers. MPEG-21 UD specifies standard descriptions for a given user, her context and available services. In addition MPEG-21 UD specifies standard formats for recommendations. In this way a generic provider can horizontally integrate standard descriptions belonging to different recommendation services thus possibly returning to the user richer recommendations and, likely, a better fruition.
\end{abstract}

\section{CCS Concepts}

-Information systems $\rightarrow$ Recommender systems; Personalization; •Applied computing $\rightarrow$ Information integration and interoperability; •General and reference $\rightarrow$ Computing standards, RFCs and guidelines;

\section{Keywords}

personalization, interoperable recommendation engines, crossdomain recommender systems, knowledge integration, MPEG21 User Description

\section{INTRODUCTION}

In order to address (at least partially) information overload issues, in recent years recommender systems have been widely adopted. Nevertheless, the accuracy of recommendations a user currently receives might strongly affect the quality of her experience [2]. Then, a generic user can jointly engage more than one service provider within her ordinary fruition. These providers, in the attempt of satisfying user needs, possibly return distinct recommendations, namely recommendations not mutually related and restricted to spe- cific and separated domains (e.g. Netflix suggests movies or TV series, Youtube suggests music songs, etc). Eventually, the user might receive redundant recommendations thus having a not fully satisfactory experience. The opportunity of logically and properly integrating recommendations of items from different domains and provided by different services can enhance recommending accuracy and improve the user experience. A generic mediation mechanism for integrating user modeling data was proposed in [1]. The mediation mechanism is aimed at facilitating interoperability between recommender systems thus providing more complete and usable recommendations to the users. The MPEG-21 User Description standard (MPEG-21 UD) [4] aims at ensuring such interoperability in a standardized way. MPEG-21 UD does not standardise the way in which recommendation engines work, i.e. the specific technique adopted (e.g., collaborative and/or content-based filtering) to produce the recommendations, instead it defines standard descriptions about a given user, her context, the services and/or the items she has enjoyed and other information that can be relevant for the purpose of recommendation. In particular, MPEG-21 UD defines standard descriptions of the recommended items thus serving for a cross-domain and cross-service integration of recommendations provided by different stakeholders. This integration is believed to be crucial for improving both the accuracy of recommendations and the user experience.

The paper is organized as follows. $\S 2$ outlines the MPEG21 UD standard. $\S 3$ briefly describes two examples of use cases, namely a web based service involving news recommendations, and a personalized radio based on the new EBU hybrid content radio concept. $\S 4$ concludes the paper with final remarks.

\section{MPEG-21 UD}

A brief description of MPEG-21 UD is here provided. Currently, the standard is in the Draft International Standard (DIS) status. In $\S 2.1$ the formats and the interfaces across which the different and separate components exchange information are shown. In $\S 2.2$ the interoperability between different Recommendation Engines is highlighted.

\subsection{Conceptual model}

MPEG-21 UD aims at enabling the horizontal integration of Recommendation Services that provide standard, i.e. com-
ACM 978-1-4503-3738-0.

http://dx.doi.org/10.1145/xxxx.x 


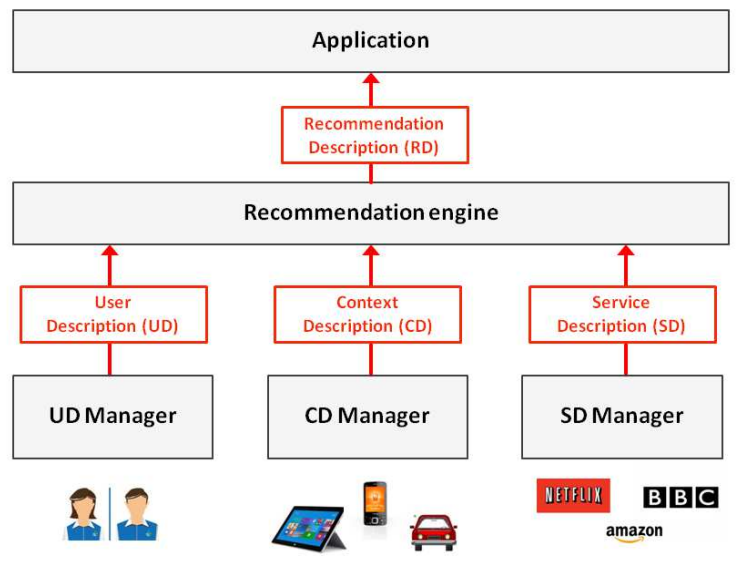

Figure 1: Conceptual Model of MPEG-21 UD. The formats specified are UD/CD/SD/RD (red). Managers, Recommendation Engine and Application (black) are out of the scope of the standard.

patible, recommendations. By a proper integration of standard recommendations, a generic service provider can return to the user a richer and enhanced fruition thus greatly matching her needs. To achieve this goal, MPEG-21 UD standardizes the input and output formats of a generic Recommendation Engine. It must be noticed that, the technologies implemented by this Recommendation Engine are out of the scope of MPEG-21 UD. As shown in Fig.1, the input formats include: a Description of the User (UD) seeking recommendation; a Description of the Context (CD) in which the User operates; a Description of Services (SD) offering content sought by User. The output format, called Recommendation Description (RD), includes: subsets of UD/CD/SD which are the actual recommended items together with information about what role these items play in the recommendation; additional logical relations linking recommended items (e.g. a ranked list specifying a preference order of the recommended items and other similar structures); descriptive metadata related to the recommended items expressed using MPEG-21 DIDL mechanism [3].

The User Description (UD) is a set of descriptions containing information about the user. This information can be either static (e.g. user's birthdate) or dynamic (e.g. the history of the user's interactions, preferences, security settings).

The Context Description (CD) is a set of descriptions of the environmental situation in which the user operates (e.g. user's device in use, physical position, temperature, humidity, sound level).

The Service Description (SD) is a set of descriptions including information about the service (or a set of subservices), that is offered to the end-user application (e.g. music tracks on demand, newscasts) and the objects (e.g. multimedia items) offered by the service.

The Recommendation Description (RD) is a set of rec-

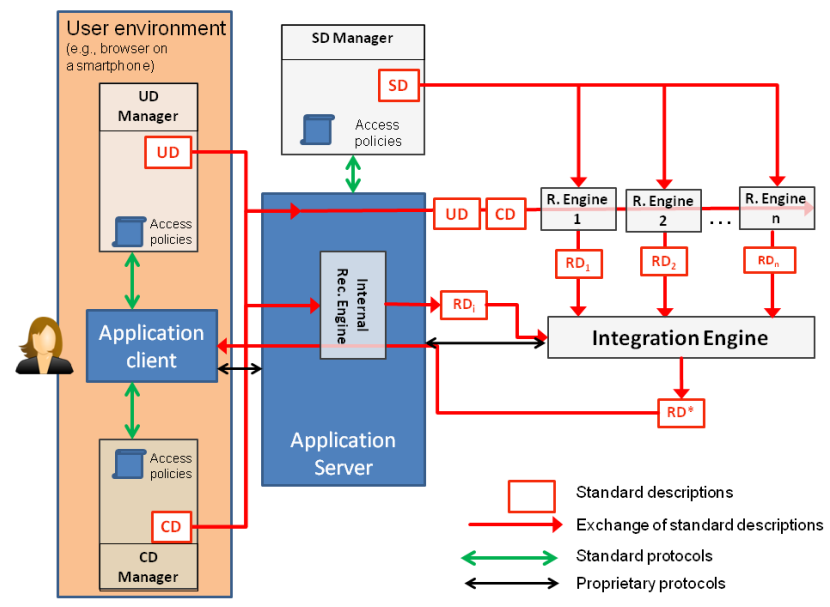

Figure 2: MPEG-21 UD ensures the interoperability between different Recommendation Engines.

ommended items provided in a structured form. RD includes the recommended objects and the services by which they are provided (e.g. an audio interview provided by a broadcaster's website), descriptive information about the recommended items and additional logical relations among the recommended items.

Fig. 1 also shows several components playing specific roles in the model.

The UD/CD/SD Managers provide functionalities of filtering, accessing, storing, editing, updating and securing $\mathrm{UD} / \mathrm{CD} / \mathrm{SD}$. The implementation of these Managers is out of the scope of MPEG-21 UD. Nevertheless, their functionalities are absolutely fundamental. For example, the UD Manager can ensure the authentication/authorization of the user accessing web applications and/or IP-connected media devices. Additionally, leveraging the UD Manager, the user can actively and at any time choose of sharing (totally, partially or not at all) her personal data. She can actively and at any time choose the Service(s) Provider(s) with whom her experience can be shared. In conclusion, the UD Manager allows for dynamically implementing her personal policies.

The Recommendation Engine is a process (or a set of processes) in charge of exploiting all available information contained in UD, CD and SD to produce a compact information, i.e. RD, for the enriched user experience. The way in which Recommendation Engine works is out of the scope of the MPEG-21 UD standard.

\subsection{Interoperable Recommendation Engines}

The opportunity for a content provider of horizontally integrating standard descriptions compliant to multiple and distinct recommendation formats is here highlighted. Let a given user start her fruition with a generic Service Provider, by using a whatever device and using an application software (see Fig. 2). Without losing generality we here assume that appropriate mechanisms are put in place to ensure that the application is aware about the identity of the user, e.g. through the application of one of several authentication 
schemes and protocols existing for generic platforms. Once the application has received information about the user and her context, namely UD and CD, an external recommendation service is enquired. In fact even if the Service Provider may have its own internal Recommendation Engine, it may decide to request the auxiliary functionalities of $n$ different and external Recommendation Engines in order to enhance the user experience. According to the policies set by the user in terms of availability of her information to external applications, the Service Provider can forward UD, CD descriptions (or subsets of them) and its own SD description to these Engines. These Engines can adopt any internal logic to return to the Service Provider a set of standard recommendations, namely $\mathrm{RD}_{1}, \mathrm{RD}_{2}, . ., \mathrm{RD}_{n}$. At this point, according to any internal logic, the Integration Engine can integrate the items recommended thus returning to the user an enhanced recommendation service. According to this schema, the policies set by the user (e.g. by the UD Manager) are fundamental and can indirectly impact on the final recommendation.

\section{USE CASES}

This section is aimed at giving a general idea about how MPEG-21 UD can be adopted in real-life scenarios. Here we briefly present two simple examples of use cases without providing any technical solution regarding its implementation. It is given to the reader the opportunity of further taking into account different use-case genres.

Web-based news recommendation. The use of online news is an extremely popular phenomenon. However, the availability on a daily basis of a wide variety of information sources generates a disproportionately high amount of texts and videos that makes it impossible for users to read and/or watch everything that is published. News content recommendation aims at satisfying specific user's news interests, while minimizing efforts in searching for the desired information. MPEG-21 UD can be effectively adopted to simplify the integration and interoperability of news recommendation services across the Web. Leveraging standard descriptions, the recommendation can be tailored by taking into account the overall fruition a user has had on different services.

Hybrid Content Radio. The second use case is more related to broadcast media. In the last years broadcasters have been witnesses of a disruptive transition from a linear programming to a world of internet-delivered personalized or on-demand contents. Personalized audio streaming services are continuously gaining popularity. Pandora, Spotify and Deezer are some of the most popular services that provide streaming contents fully customized on listener preferences. Hybrid content radio (HCR, see [5]) aims at making broadcast radio more flexible, allowing to replace part of the broadcast audio content with personalized audio from the Internet. HCR manages all the 'intermediate scenarios' where the broadcaster provides a linear programming and, on the other side, recommends enriching and context-based online content. Leveraging MPEG-21 UD descriptions, the content can be personalized for a specific listener: based on the description of the user and of her history, a recommender system can hint alternative audio content that will replace part of the broadcast content thus increasing her satisfaction and decreasing her tendency to switch channels. MPEG21 UD allows to leverage additional information derived by services used by the same listener on different platforms, increasing the base of information for the recommender system.

\section{CONCLUSIONS}

To date, the user fruition can be strongly affected by vertical and possibly redundant recommendations coming from different service providers. Furthermore, in order to improve accuracy and appropriateness of cross-domain recommendations, standard descriptions about a given user and her context are needed, as well as interoperability between different recommendation results. In this paper we have illustrated how the emerging standard MPEG-21 UD addresses these issues: by allowing for the integration of recommendations provided by different engines, and, by defining standard descriptions for the users, their context and the services providing potentially interesting (i.e. recommendable) items. Adopting MPEG-21 UD standard descriptions, a service provider can properly integrate multiple recommendations thus enhancing its service and, likely, improving the user experience. Two simple examples of use cases, namely a web based service involving news recommendations, and a personalized radio based on the hybrid content radio concept have been described to further illustrate the concept.

\section{REFERENCES}

[1] S. Berkovsky, T. Kuflik, and F. Ricci. Mediation of User Models for Enhanced Personalization in Recommender Systems. User Modeling and User-Adapted Interaction, 2008.

[2] Francesco Ricci, Lior Rokach, Bracha Shapira. Introduction to Recommender Systems Handbook, chapter 1, pages 1-35. Springer, 2011.

[3] ISO/IEC 21000-2:2005. Information technology Multimedia framework (MPEG-21) - Part 2: Digital Item Declaration, 2005.

[4] ISO/IEC DIS 21000-22: Information technology Multimedia framework (MPEG-21) - Part 22: User Description. 2015.

[5] P. Casagranda, A. Erk, S. O'Halpin, D. Born and W. Huijten. A Framework for a Context-Based Hybrid Content Radio. In International Broadcasting Convention (IBC), Amsterdam, 2015. 